\title{
Mutations affecting stability and deadenylation of the yeast MFA2 transcript
}

\author{
Denise Muhlrad and Roy Parker ${ }^{1}$ \\ Department of Molecular and Cellular Biology, University of Arizona, Tucson, Arizona 85721 USA
}

\begin{abstract}
Decay rates of individual mRNAs in the yeast Saccharomyces cerevisiae can vary by 10- to 20 -fold. To determine the basis for the rapid degradation of the mRNA encoded by the yeast MFA2 gene we have used a genetic screen to isolate mutations that increase the stability of this transcript. Analysis of point mutations obtained from this screen, and of additional lesions constructed in vitro, indicated that the MFA2 3 '-untranslated region (UTR) contains sequences that specify rapid mRNA decay. Moreover, the lesions that affected mRNA decay rate also affected the process of mRNA deadenylation. Mutations in one region of the $3^{\prime}$ UTR both decreased the rate of poly(A) shortening and increased the stability of an intermediate form in the decay pathway with an oligo(A) tail ( $\sim 10$ nucleotides). Mutations in a second region primarily increased the stability of the oligo(A) form. These results suggest that the decay of the MFA2 mRNA initiates with the shortening of the poly(A) tail and there are specific sequences within the $3^{\prime}$ UTR that stimulate poly(A) tail shortening as well as subsequent steps in the decay pathway. Given the similarity of this decay pathway to that seen for some mammalian mRNAs, these results suggest that mRNA deadenylation may be a common mechanism of mRNA turnover.
\end{abstract}

[Key Words: Yeast MFA2 gene; 3' UTR; mRNA decay; deadenylation; poly(A) tail]

Received June 16, 1992; revised version accepted August 4, 1992.

Differences in mRNA decay rates can be a significant factor in determining both the level and the regulation of eukaryotic gene expression (for review, see Cleveland 1989; Higgins 1991; Peltz et al. 1991). Critical to an understanding of cytoplasmic mRNA turnover will be a description of the features of individual mRNAs that dictate their inherent decay rates and a mechanistic understanding of how these features affect mRNA degradation. By analogy to prokaryotic systems (for review, see Higgins 1991), differences in decay rates for eukaryotic mRNAs could arise both by the inclusion of sequences that stimulate either general or specific turnover pathways, and by the presence of features that inhibit general mRNA degradation pathways. One line of evidence for sequences that stimulate mRNA decay pathways has come from the construction of chimeric mRNAs derived from stable and unstable mRNAs. These experiments have led to the identification of a small number of specific sequence elements that can be demonstrated to confer rapid mRNA turnover when transferred to stable mRNAs. These "instability elements" can be found both in coding and in $3^{\prime}$-untranslated regions (UTRs) (for review, see Peltz et al. 1991).

Recent observations suggest that instability elements

${ }^{1}$ Corresponding author. operate in at least two ways. One mechanism of transcript destabilization appears to be coupled to the removal of the poly(A) tail. Analysis of the decay of transcripts containing the so-called AU-rich instability element, which is found in the 3' UTR of some mammalian mRNAs, such as those encoded by the proto-oncogenes $c-f o s$ and c-myc, suggests that the first step in the decay of these mRNAs is deadenylation (Brewer and Ross 1988; Shyu et al. 1991; Swartwout and Kinniburgh 1991). In this mechanism, one of the functions of the AU-rich element appears to be to stimulate the rate of poly $(\mathrm{A})$ tail shortening (Wilson and Triesman 1988; Shyu et al. 1991). An alternative pathway, based on the detection of mRNA fragments, is that other instability elements contain endonucleolytic cleavage sites (Binder et al. 1989; Brown and Harland 1990). The generality of these mechanisms and whether or not deadenylation is a prerequisite for some internal cleavages is unclear.

The genetic approaches possible in the yeast Saccharomyces cerevisiae make this organism a useful system for the analysis of eukaryotic mRNA decay. Previously, we have reported the decay rates of a large number of yeast mRNAs, including both stable and unstable mRNAs (Herrick et al. 1990). One class of mRNAs examined were those regulated by mating type. Many of these mRNAs are unstable, possibly because they need to be removed rapidly from the cell after mating-type 
switching. Our analysis of one such mRNA, that encoded by the MAT 1 gene, has identified sequences within the coding region that are involved in stimulating rapid mRNA decay (Parker and Jacobson 1990).

The mRNA produced from the MFA2 gene is unstable (Herrick et al. 1990) and encodes the mating pheromone, a-factor (Michaelis and Herskowitz 1989). Because of the extremely short coding region of this RNA (114 nucleotides) and the relatively long 3' UTR (169 nucleotides), it seemed plausible that the sequences responsible for its rapid decay would be localized in the $3^{\prime}$ UTR and, therefore, would represent a different type of instability element from that in the $M A T \alpha 1$ transcript. In this paper we describe our analysis, using both an in vivo screen and in vitro constructions, of features within the MFA2 mRNA that stimulate its decay. This analysis indicates that the 3' UTR is involved in stimulating mRNA decay. Moreover, lesions that affect mRNA decay rate also affect either the rate of poly(A) tail shortening or the stability of an intermediate form in the decay pathway with an oligo(A) tail, or both. These results suggest that the decay of the MFA2 mRNA initiates with the removal of the poly(A) tail and that there are specific sequences within the $3^{\prime}$ UTR that stimulate distinct steps in the deadenylation process. Importantly, the similarity of this decay pathway to that described for some mammalian mRNAs suggests that this mechanism of mRNA turnover may be a common decay pathway.

\section{Results}

\section{Strategy}

The approach that we have used to identify mutations that inhibit turnover of the MFA2 transcript is illustrated in Figure 1. The cornerstone of this strategy is that mutations that increase the half-life of the MFA2 mRNA should raise its steady-state level and, therefore, lead to higher levels of expression of the gene product, a-factor. Because a-factor arrests the growth of $\alpha$ cells in the $G_{1}$ phase of the cell cycle, the levels of a-factor produced can be assayed in a semiquantitative manner in what has been termed a halo assay (see Michaelis and Herskowitz 1989). In this assay the level of a-factor determines the size of growth inhibition in a lawn of $M A T \alpha$ cells around a colony of MATa cells (Fig. 1). Using this assay we have screened $\sim 1800$ mutants generated by PCR mutagenesis followed by in vivo recombination (Muhlrad et al. 1992) to identify mutations that overexpress a-factor (for details, see Materials and methods).

Point mutations in the $3^{\prime}$ UTR affect the decay rate of the MFA2 transcript

To determine whether the increased a-factor production was the result of a change in the decay rate of the MFA2 mRNA, the half-lives of the MFA2 transcripts in 11 overexpressing mutants were measured using a temperaturesensitive mutation in RNA polymerase II (Herrick et al. 1990; Parker et al. 1991). Eight of the mutations caused

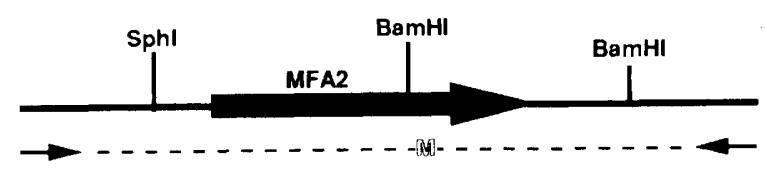

1. MUTAGENIZE MFA2 GENE BY PCR

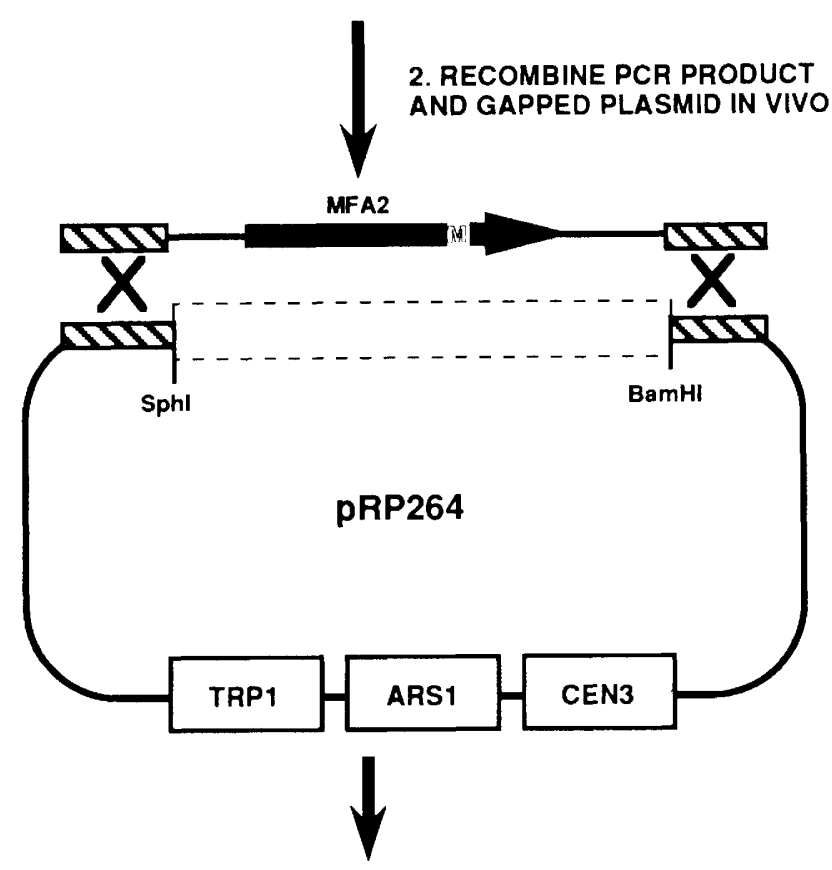

3. HALO ASSAY OF a FACTOR LEVELS

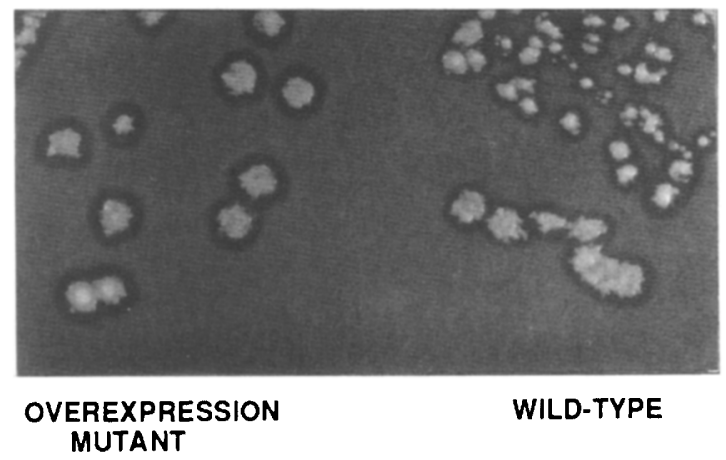

Figure 1. Mutagenesis strategy. Shown is the basic approach used to isolate mutations within the MFA2 gene that lead to increased expression of a-factor. The hollow $M$ is used to signify a mutation induced during error-prone PCR. (Middle) The hatched boxes represent regions of homology between the PCR product and the gapped plasmid. (Bottom) A section of a plate showing the a-factor halos of an overexpression mutant and the wild type on a lawn of a MAT $\alpha$ strain, SM1086, with increased sensitivity to mating pheromones owing to the sst2 mutation.

no change in decay rate or steady-state levels of the $M F A 2$ transcript and led to overexpression of a-factor for reasons unrelated to mRNA levels (data not shown). As shown in Figure 2, three of the mutants increased the half-life of the transcript. The half-lives of these mutants differed from the wild-type value of $3.5 \mathrm{~min}$ to half-lives 


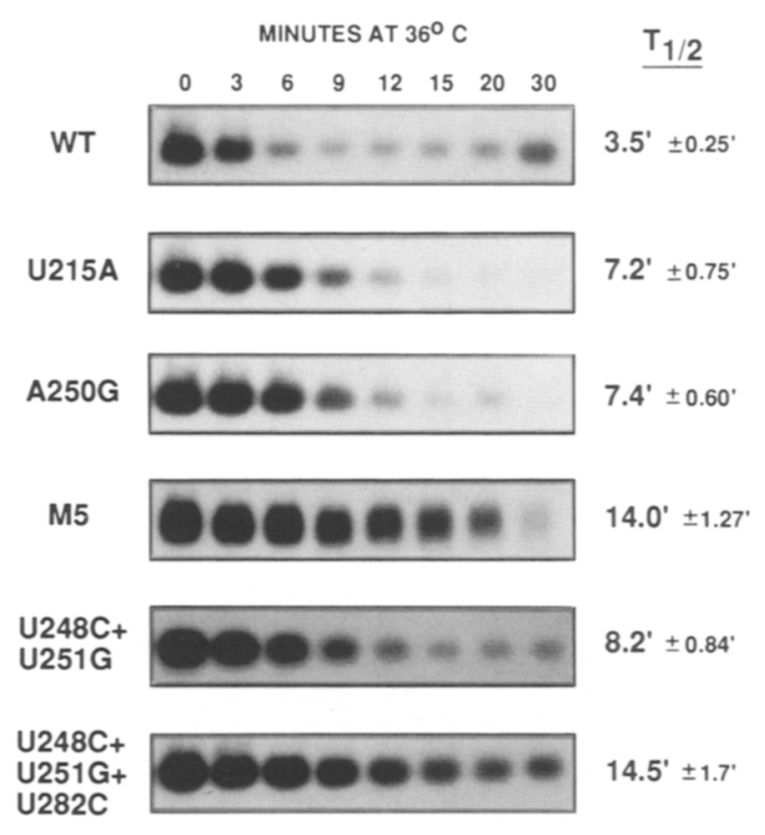

Figure 2. mRNA decay rates of MFA2 mutants. Shown are Northern blot analyses of the wild-type MFA2 transcript and transcripts with various point mutations that affect mRNA decay rates. The mutation present in each experiment is shown at left. The Northern blot shown for the M5 mutant is as originally isolated and contains five mutations: U231C, U240G, U248C, U251G, and U282C. The last two panels show Northern blots of the relevant combinations of point mutations within the M5 mutant that affect mRNA decay. The probe for these blots is a random-primed SphI-NdeI fragment containing the MFA2 gene. The increase in signal seen in the wild-type mRNA, at late time points, results from an accumulation over time, beginning at $\sim 25$ min after shift to high temperature, of mRNAs with long poly(A) tails. This may be the result of a secondary consequence of inhibition of transcription leading to a defect in either poly|A| shortening or nuclear-cytoplasmic transport, thus allowing the residual transcription in the temperature sensitive $r p b 1-1 \mathrm{mu}-$ tant $\{\sim 5 \%$ of wild-type levels $)$ to eventually accumulate these species.

ranging from $7.2-14 \mathrm{~min}$. Similarly, these three mutations led to a corresponding increase in steady-state levels of MFA2 mRNA and a-factor protein levels, as judged by a dilution assay (see Materials and methods; data not shown). The correlation between mRNA and protein levels argues that there are no major differences in the translational efficiency of the different MFA2 transcripts.

The entire $M F A 2$ gene from each of the mutants with decreased decay rates was sequenced. Two mutants, $\mathrm{U} 215 \mathrm{~A}\left(t_{1 / 2}=7.2 \mathrm{~min}\right)$ and A250G $\left(t_{1 / 2}=7.4 \mathrm{~min}\right) \mathrm{had}$ unique changes (mutations are named by listing the wild-type base, the nucleotide position within the MFA2 transcript, and the change in the mutation). The M5 mutant $\left(t_{1 / 2}=14 \mathrm{~min}\right)$ carried five base changes (U231C, U240G, U248C, U251G, and U282C). To determine which of these base changes was responsible for the observed effect on mRNA decay rate, we created by oligo- mutagenesis the mutations U231C, U240G, U248C, U251G, and U282C separately in an otherwise wild-type MFA2 gene. The effect of any single mutation was minimal (summarized in Fig. 4). Subsequent analysis of various mutant combinations showed that a partial effect on mRNA half-life results from the combination of the U248C and U251G mutations (double mutant $t_{1 / 2}=8.2$ min; Fig. 2, fifth panel). The full M5 phenotype $\left(t_{1 / 2} \sim 14\right.$ $\mathrm{min}$ ) then results from the addition of the point mutant U282C to this combination (triple mutant $t_{1 / 2}=14.5$ min; Fig. 2, bottom panel).

\section{Deletion analysis identifies a region required for rapid decay}

The observation that combinations of point mutations led to slower decay rates than individual changes suggested that the sequences within the MFA2 3' UTR involved in stimulating mRNA degradation were complex and/or partially redundant in function. To further this analysis we constructed several linker-scanning mutations and then used these sites to create a set of $3^{\prime}$ UTR deletions. The linker-scanning mutations include four BglII sites and an XhoI site (shown schematically in Fig. 3). Because these sites involve multiple changes, each site is designated by either a B (for BgIII) or an X (for XhoI) followed by the position of the first nucleotide in the resulting restriction site. The exact changes are shown in Figure 4. As summarized in Figure 4, the creation of these sites has at most a minimal effect on mRNA decay. This result indicates that none of the 19 nucleotides altered in these sites are required to stimulate rapid decay.

These restriction sites were then used to create the deletions shown in Figure 3. Deletion of the region between nucleotides 184 and $241, \Delta \mathrm{B} 178-241$, increases the half-life roughly threefold $\left(t_{1 / 2}=9.5 \mathrm{~min}\right.$; Fig. 3 ; see legend for description of deletion nomenclature). Smaller deletions within this region, $\Delta \mathrm{B} 178-207, \Delta \mathrm{B} 178-222$, and $\Delta B 207-241$, also increase the stability of the transcript, although the effect is relatively minor (Fig. 3 ) in some cases (e.g., $\Delta B 178-207$ ). These results led us to conclude that the region between 184 and 241 is required for rapid decay. The observations that smaller deletions have intermediate effects suggests that the functional feature in this region is not simple and may be the result of a particular structure involving the entire region (see Discussion). For discussion purposes we refer to this region, nucleotides $184-241$, as region $\mathrm{I}$.

\section{A second region of the MFA2 3' UTR can also affect mRNA turnover}

Deletion of nucleotides between the $X$ hoI site created at position 245 , X245, and a naturally occurring $N d e I$ site at position $288, \Delta \mathrm{X} 245-288$, had no effect on decay rate $\left(t_{1 / 2}=3.7 \mathrm{~min}\right.$; Fig. 3). This result demonstrated that this region is not essential for rapid decay. A larger deletion including these sequences and region I (nucleotides 184-241), $\Delta \mathrm{B} 178-288$, however, resulted in a small but reproducible increase in stability ( $\Delta \mathrm{B} 178-288$, 


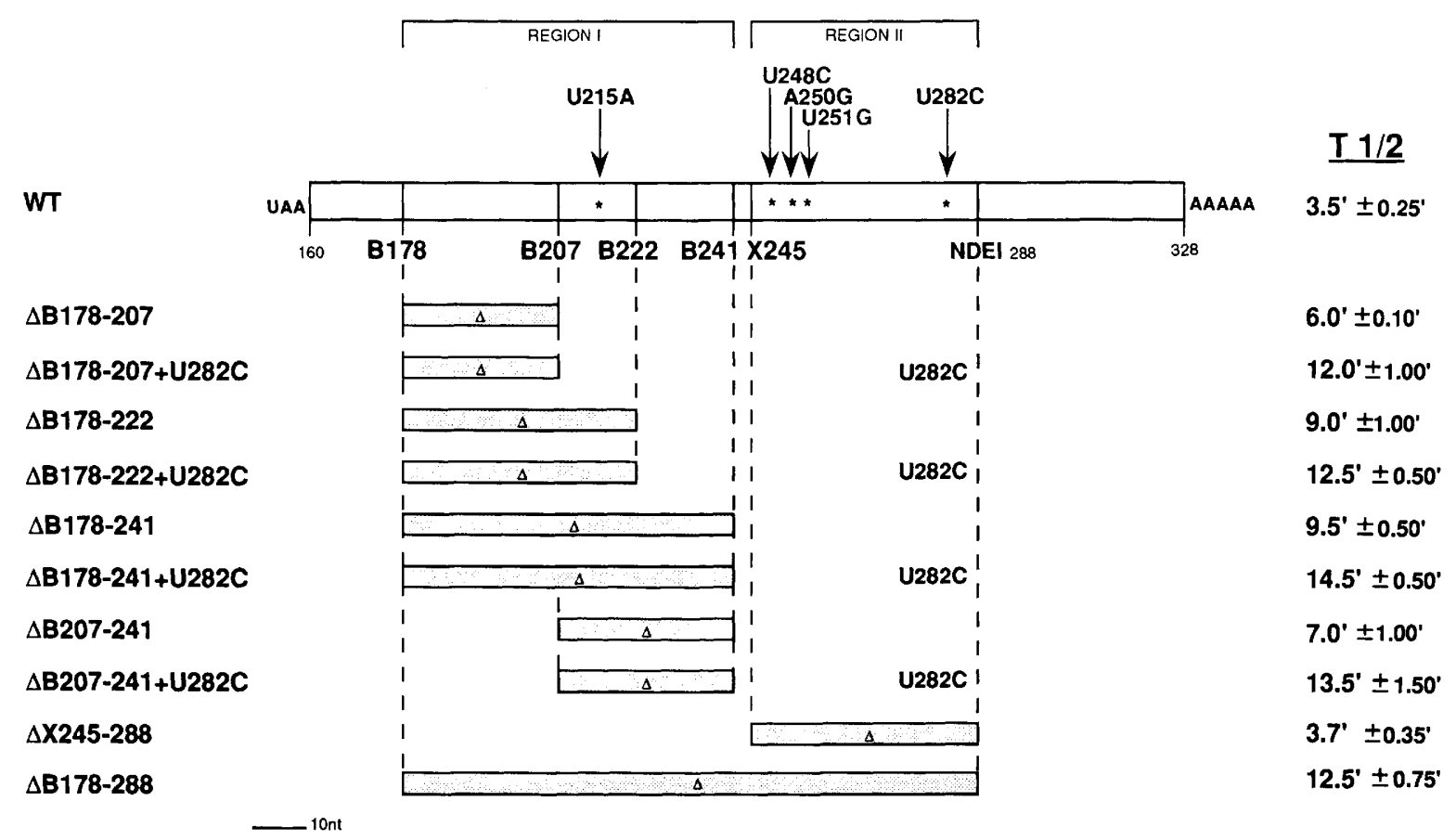

Figure 3. Effects of $3^{\prime}$ UTR deletion analysis on MFA2 transcript decay. Shown are the positions of deletions within the MFA2 $3^{\prime}$ UTR and the decay rate of the corresponding transcript. Except for $\triangle \mathrm{B} 178-288$ and $\triangle \mathrm{X} 245-288$, mRNA decay rates were determined in the presence and absence of the U282C mutation. Deletions were constructed from sites created by oligomutagenesis. These sites shown above the schematic are B178, B207, B222, and B241, which are BgIII sites, X245, an XhoI site, and a naturally occurring NdeI site (see Fig. 4). Each deletion is named by giving the $5^{\prime}$ site from which the deletion was constructed and the position of the site at the $3^{\prime}$ boundary. For example, $\Delta \mathrm{B} 178-241$ is a deletion between the BgIII site at nucleotide 178 and the $B g I I I$ site at position 241 . The shaded boxes represent the deleted sequences. Relevant point mutations that were isolated in our genetic screen, as well as the locations of the regions referred to as regions I and II, are also shown above the schematic. The $\Delta \mathrm{B} 178-288$ and $\Delta \mathrm{X} 245-288$ deletions affected the accuracy of 3 '-end formation. For this reason, the decay rates of these transcripts were determined by resolving the transcripts with the proper $3^{\prime}$ end from longer aberrant transcripts on acrylamide Northern blots and only considering the properly terminated species in our calculations. For each mutation, the given decay rate is the average of multiple measurements.

$\left.t_{1 / 2}=12.5 \mathrm{~min}\right)$ as compared with the deletion of region I alone ( $\Delta \mathrm{B} 178-241, t_{1 / 2}=9.5 \mathrm{~min}$ ). (We were unable to analyze the effects of deletions extending $3^{\prime}$ of position 288 because such deletions abolished the production of properly terminated mRNAs.) These results suggested that although the sequences between nucleotides 245 and 288 are not required for rapid decay, this region can contribute to decay in the absence of region I. For discussion purposes we refer to this region, nucleotides $245-288$, as region II.

These results are similar to those seen with the original point mutations, wherein the largest effects on mRNA turnover in the M5 mutant resulted from a combination of the U248C, U25IG, and U282C point mutations. Given this we examined the effect of the U282C mutation on other alterations in region $I$. The results of such experiments [summarized in Fig. 3, Table 1, and see below (Fig. 6)] indicated that in each case the addition of the U282C mutation to other lesions increased the stability of the transcript. These experiments also point out that some region I mutations, which have small effects on their own (e.g., B222, $t_{1 / 2}=5.5 \mathrm{~min}$ ), have hindered region I function, as judged by the synergistic effect of the U282C mutation on mRNA decay (e.g., B222 +
U282C, $t_{1 / 2}=12.5 \mathrm{~min} /$. These observations argue that when region I is altered, region II can function to stimulate mRNA decay, albeit at a reduced rate when compared with region $\mathrm{I}$.

These results, in combination with those discussed above, suggested that the $3^{\prime}$ UTR of MFA2 has two regions that are involved in stimulating mRNA decay. The first, termed region I, is located between nucleotides 184 and 241 and can function in the absence of region II to stimulate decay at a rate similar to that of the normal MFA2 transcript. Region II, located between nucleotides 245 and 288, is not required for rapid decay but, in the absence of region I, can still function to stimulate decay, albeit less efficiently. Alteration of both regions results in a four- to fivefold increase in stability compared with the parental MFA2 transcript.

\section{Region I is sensitive to secondary structure} in the $3^{\prime}$ UTR

One of the surprising features of our analysis was a set of point mutations that affected mRNA decay located between positions 248 and 252. These mutations include A250G, the double mutant U248C + U251G, and a qua- 

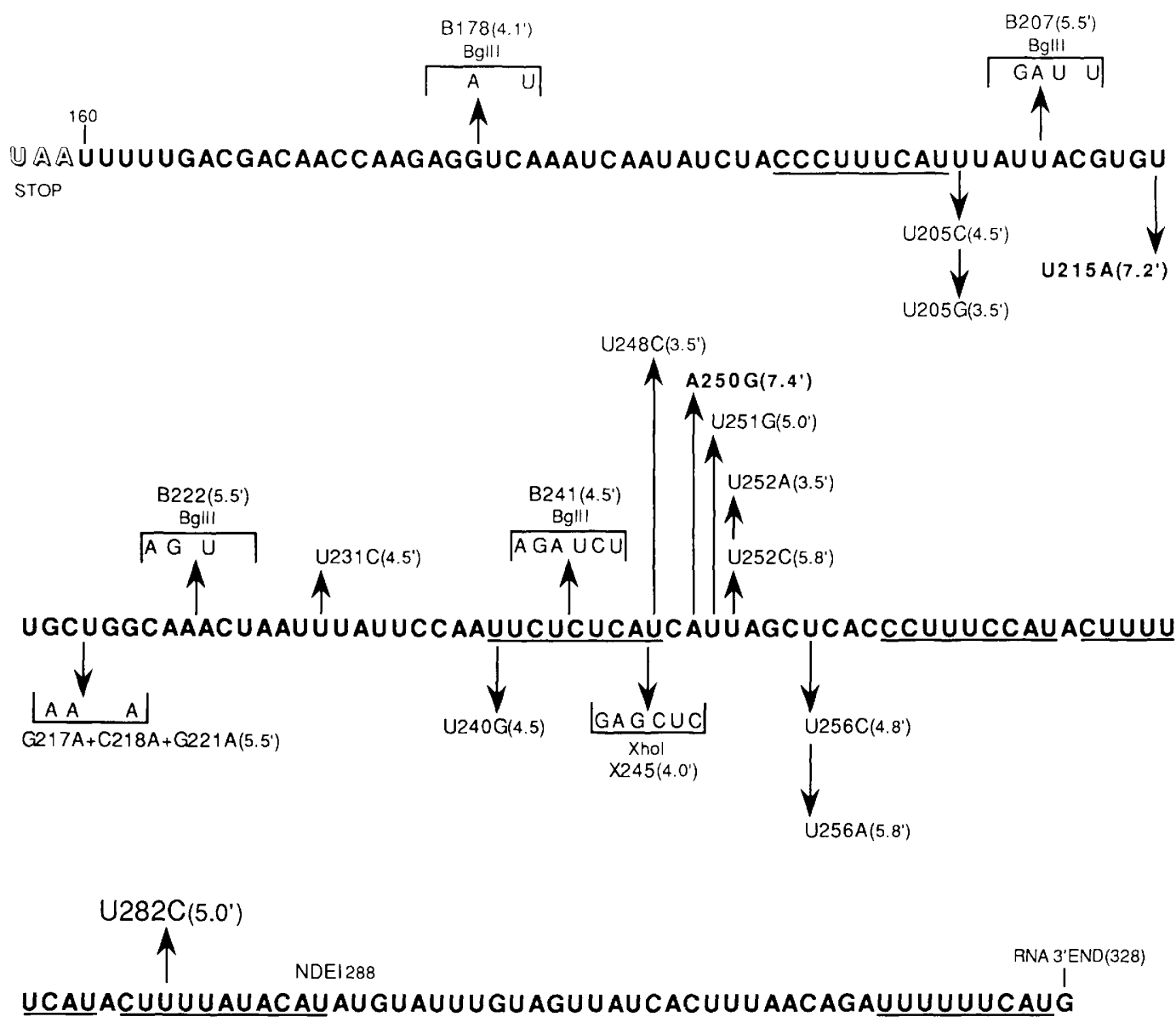

Figure 4. Point mutations in the $3^{\prime}$ UTR and mRNA decay rates. Shown is the sequence of the MFA2 $3^{\prime}$ UTR from the first base after the termination codon (position 160) to the major end of the mRNA (position 328; A. Sachs, pers. comm.). Point mutations are shown above and below the wild-type sequence, with the resulting mRNA decay rate shown in parentheses. Decay rates are all the average of two or more measurements for each mutation with standard deviations that are typically $<10 \%$ (see Table 1). The restriction sites created are also illustrated in the same manner. Mutations that involve multiple changes are shown within brackets. The repeated sequence motifs discussed in the text are underlined.

druple mutant we synthesized U248C + U251G + $\mathrm{U} 252 \mathrm{C}+\mathrm{U} 256 \mathrm{C}$. Although these point mutations increase the stability of the mRNA, replacement of a portion, X245 (Fig. 4), or deletion of the region in which they were located, $\Delta X 245-288$ (Fig. 3), had no effect on mRNA turnover. One explanation of this apparent paradox was that the mutations that affect mRNA decay do so by creating new inhibitory RNA secondary structures. Computer analysis using the stem-loop program of the GCG computer software package indicated that the mutations in this region that affected mRNA decay all led to the creation of new helices with $>8$ contiguous bp. For example, the double mutant, U248C + U251G, and the quadruple mutant, $\mathrm{U} 248 \mathrm{C}+\mathrm{U} 251 \mathrm{G}+\mathrm{U} 252 \mathrm{C}+$ $\mathrm{U} 256 \mathrm{C}$, both increase the stability of the 8 base stem shown in Figure 5. In contrast, mutations with no effect on mRNA decay, such as B241, X245, U252A, and $\Delta \mathrm{X} 245-288$, did not promote any such structure.

If the effect of these mutations on mRNA turnover results from a new inhibitory secondary structure, then compensatory mutations that disrupt the predicted stem should restore the mRNA to rapid decay, provided the sequences that make up the $5^{\prime}$ part of the stem are not themselves required for rapid decay. To examine this possibility, we combined the quadruple mutant (U248C $+\mathrm{U} 251 \mathrm{G}+\mathrm{U} 252 \mathrm{C}+\mathrm{U} 256 \mathrm{C}$ ) with a change of three bases, G217A + C218A + G221A, in the 5' portion of the stem predicted to disrupt the helix (see Fig. 5). Computer estimations of the effect of these changes predicts a folding energy of $-8.0 \mathrm{kcal}$ for the quadruple mutant alone as compared with a folding energy of $-0.1 \mathrm{kcal}$ when combined with the $5^{\prime}$ changes. The disruption of the stem largely suppresses the effect on mRNA decay rate of the quadruple mutant $\left(t_{1 / 2}=8.2\right.$; see Fig. 5$)$ by decreasing the half-life to $4.5 \mathrm{~min}$. This result argues that the major effect of these mutations was to stabilize this helix, which is inhibitory to mRNA decay. These results also indicate that none of the three bases changed in the compensatory mutant is critical for region I function. 
Table 1. mRNA decay rate for point mutant combinations

\begin{tabular}{lr}
\hline Point mutant combinations & $t_{1 / 2}$ \\
\hline Double mutants & \\
U205C + U231C & $4.5^{\prime} \pm 0.5^{\prime}$ \\
U240G + U282C & $7^{\prime} \pm 0^{\prime}$ \\
U231C + U240G & $4^{\prime} \pm 0^{\prime}$ \\
U248C + U251G & $8.17^{\prime} \pm 0.84^{\prime}$ \\
Triple mutants & \\
U248C + U251G + U231C & $8.25^{\prime} \pm 0.75^{\prime}$ \\
U248C + U251G + U282C & $14.5^{\prime} \pm 1.7^{\prime}$ \\
Quadruple or more mutants & \\
M5[U231C + U240G + U248C & \\
+ U251G+ U282C] & $14^{\prime} \pm 1.27^{\prime}$ \\
U248C + U251G + U252C + U256C & $8.2^{\prime} \pm 0.8^{\prime}$ \\
U248C + U251G + U240G + U231C & $8^{\prime} \pm 0^{\prime}$ \\
B207 + U282C & $7^{\prime} \pm 1^{\prime}$ \\
B222 + U282C & $12.5^{\prime} \pm 2.5^{\prime}$ \\
\hline
\end{tabular}

Half-lives are the average of multiple experiments with standard deviations as shown. B207 is the combination of U208G + $\mathrm{U} 209 \mathrm{~A}+\mathrm{A} 210 \mathrm{U}+\mathrm{G} 212 \mathrm{U}$ (see Fig. 4). B222 is the combination of $\mathrm{C} 222 \mathrm{~A}+\mathrm{A} 223 \mathrm{G}+\mathrm{A} 225 \mathrm{U}$ (see Fig. 4).

We have also examined the effects of the compensatory mutations G217A + C218A + G221A when introduced into an otherwise wild-type $3^{\prime}$ UTR. In this case, the transcript can form a similar, but weaker 3 GC base pairs exchanged for $A U$ base pairs; predicted $\Delta G=-3.4$ kcal) structure. These three mutations have only a minor effect on the decay rate of the mRNA (Fig. 5, bottom panel; $t_{1 / 2}=5.3 \mathrm{~min}$ ). Moreover, the triple mutation behaves almost the same by itself as in combination with the original quadruple mutant. These results suggest that although the formation of new secondary structures can be inhibitory for decay, such stems must have a folding energy above a minimum level. This result is not unexpected, considering that the ability of a new secondary structure to form and inhibit decay will depend on its relative stability when compared with possible alternative structures that are required for decay (for analogous discussion, see Stormo 1986).

\section{Mutations that affect $m R N A$ decay also affect deadenylation}

The experiments described above allowed the conclusion that sequences within the 3' UTR of MFA2 function to stimulate decay. As discussed previously, precedent exists for sequences within the $3^{\prime}$ UTR of metazoan mRNAs functioning to stimulate mRNA decay by either containing endonucleolytic cleavage sites (Binder et al. 1989; Brown and Harland 1990) or by stimulating mRNA deadenylation (Brewer and Ross 1988; Wilson and Triesman 1988; Shyu et al. 1991; Swartwout and Kinniburgh 1991). If degradation proceeds by endonucleolytic cleavage we might be able to detect the initial intermediates as has been seen in other cases. We examined long exposures of Northern blots of the MFA2 transcript and found no evidence for specific breakdown products. We estimate that we would have been able to detect them if they were present at $5 \%$ of the mature mRNA levels and had a half-life of 10-20 sec.

To determine whether the sequences within the MFA2 3' UTR affect the process of deadenylation, we examined the size of the poly(A) tail on the various mutant mRNAs after inhibition of transcription. In these experiments we used polyacrylamide Northern blots that allow for resolution of different poly(A) tail lengths. As can be seen in Figure 6, the steady-state population of MFA2 transcripts is heterogeneous with a range of $\sim 10-80 \mathrm{nu}-$ cleotides. Because nascent MFA2 transcripts have a narrow distribution of long poly(A) tails, this distribution reflects a population of transcripts at different stages in the deadenylation process (data not shown). This range is consistent with what would be expected from prior examination of the population of poly(A) tails in yeast (Groner et al. 1974). After inhibition of transcription by a shift to high temperature, the poly(A) tail shortens rapidly on wild-type transcripts. We estimated the rate of poly(A) shortening by determining the length of the longest poly(A) tails in the population, which correspond to the newly synthesized transcripts at zero time, as a function of time. Using this analysis on four separate experiments, we calculated that the rate of poly(A) shortening of the wild-type MFA2 transcript is $\sim 11-14$ adenosines per minute. Note that the poly $(A)$ tail never shortens to zero as judged by comparison with a sample in which the poly(A) tail has been removed by cleavage with RNase $\mathrm{H}$ in the presence of oligo(dT) (left lane in all panels). The shortest poly(A) tail detected has a length of 10-12 adenylate residues.

Mutations in region I affect the deadenylation process We have examined the effects of the mutations within domain I on poly(A) tail shortening. All of these mutations decrease the rate of shortening of the poly $(A)$ tail as judged by the persistence of mRNAs with long poly(A) tails in the population for increased lengths of time compared with the wild-type transcript (Fig. 6; data not shown). Examples of this effect can be seen in Figure 6 by comparing A with B, C, D, and E. In multiple experiments, measuring the longest poly(A) tails for each mutant transcript as a function of time, we estimate the rate of poly(A) tail shortening to be between 3 and 5 adenylate residues per minute for transcripts with the $\Delta \mathrm{B} 178-241$ and U248C + U251G mutations (Fig. 6, B, C), and $\sim 6-8$ adenylate residues per minute for transcripts with the $\Delta \mathrm{B} 178-207, \mathrm{~B} 222, \mathrm{~A} 250 \mathrm{G}$, and U215A lesions (Fig. 6, D, E; data not shown). The observation that mutations that increase the half-life of the mRNA also decrease the rate of poly(A) shortening suggests that shortening of the poly(A) tail is a prerequisite in the decay of this mRNA.

The second effect of the region I lesions on the deadenylation reaction is to increase the stability of the final product observed, a form with $\sim 10-12$ adenosine residues remaining. Examples of this result are the case of the U248C + U251G double mutant (Fig. 6, C) and the $\Delta$ B178-241 deletion (Fig. $6, \mathrm{~B}$ ). Note that in these cases 
Figure 5. Effect of new secondary structures on mRNA decay. Shown are Northern blots of transcripts and the predicted possible secondary structure that can form between nucleotides 214 and 221 and nucleotides 248 and 255. A schematic of the position of this stem, illustrated as arrows, within the MFA2 $3^{\prime}$ UTR in relation to other features discussed is shown. Possible stems for each combination of mutations are shown at left. This quadruple mutant has a similar effect on mRNA deadenylation as the double mutant $\mathrm{U} 248 \mathrm{C}+\mathrm{U} 251 \mathrm{G}$, and this effect is also suppressed by the mutations that disrupt the new stem (data not shown). The mutations present are shown beside the postulated stem structure and are highlighted as hollow letters in the stem-loop cartoons.
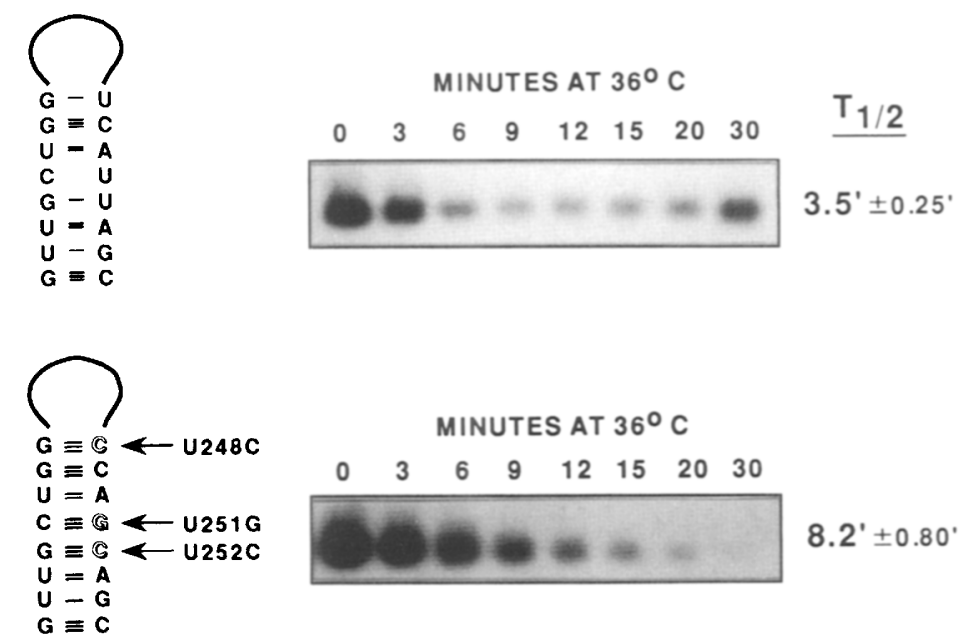

$8.2^{\prime} \pm 0.80^{\circ}$
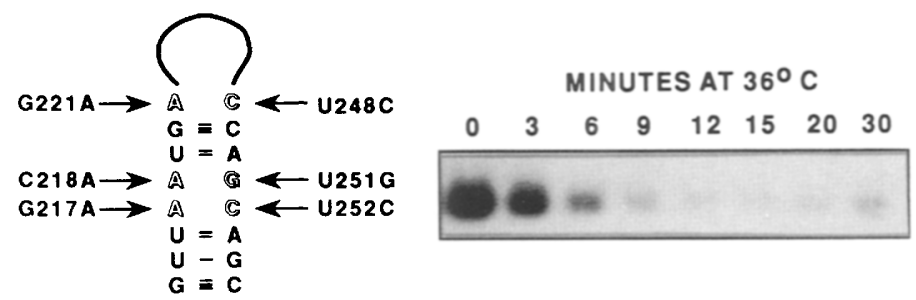

$4.5^{\prime} \pm 0.50^{\prime}$
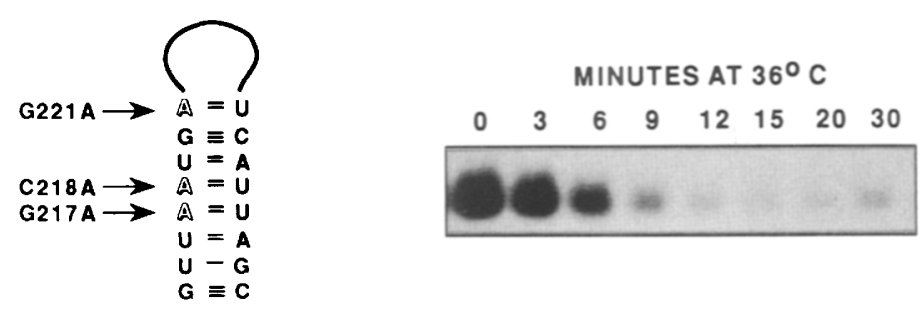

$5.3^{\prime} \pm 0.25^{\prime}$

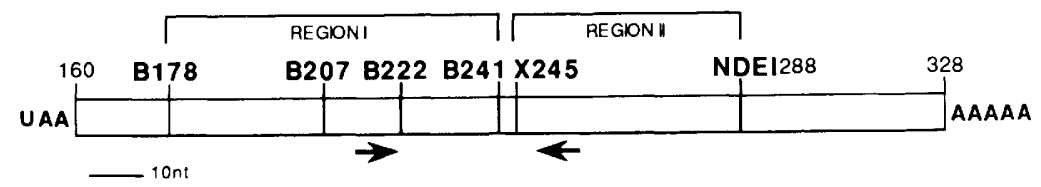

the remaining $M F A 2$ transcript is deadenylated to the oligo(A) form by $9 \mathrm{~min}$ and that this form then persists, whereas in wild-type transcripts this form disappears rapidly (Fig. 6, cf. A with $B$ and $C$ ). We can estimate the decay rates of this oligo(A) species by quantitation of its levels at time points after the depletion of the long poly(A) form. This analysis indicates that the stability of the oligo(A) form of the transcript is increased approximately twofold in these mutants [e.g., wild-type, $t_{1 / 2}$ of oligo(A) form $=2.8 \pm 0.3 \mathrm{~min}$ (Fig. $6 \mathrm{~A}$ ); $\Delta \mathrm{B} 178-241, t_{1 / 2}$ of oligo(A) form $=5.3 \pm 0.3 \mathrm{~min}$ (Fig. $6 \mathrm{~B}$ ); U248C + U251G double mutant, $t_{1 / 2}$ of oligo(A) form $=5.2 \pm 0.5$ min (Fig. $6 \mathrm{C}$ ) $\quad \Delta \mathrm{B} 207-241, \quad t_{1 / 2}$ of oligo(A) form $=5.3 \pm 0.2 \mathrm{~min}$ (Fig. $6 \mathrm{D}$ ); B222, $t_{1 / 2}$ of oligo(A) form $=5.0 \pm 0.2 \mathrm{~min}$ (Fig. $6 \mathrm{E}$ )]. The observation of a form of the mRNA persisting with an oligo(A) tail in these mutant transcripts suggests that there are at least two discrete phases in the decay of this mRNA, an initial shortening of the poly(A) tail and subsequent degradation of this oligo(A) form.

Mutations in region II increase the stability of the oligo(A) form of the MFA2 transcript As described above, mutations in region II increase the stability of mRNAs with lesions in region I. This increase in stability could either result from a further decrease in the rate of deadenylation, and/or from an additional increase in the stability of the oligo(A) form of the transcript. To examine these possibilities, we studied the effects of mutations within region II on the deadenylation reaction. The $\triangle$ X245-288 deletion has no effect on mRNA decay or deadenylation (see Fig. 3; data not shown). The only clear 
A

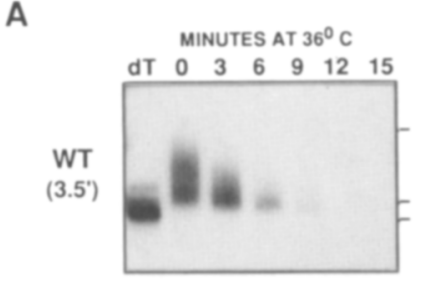

B
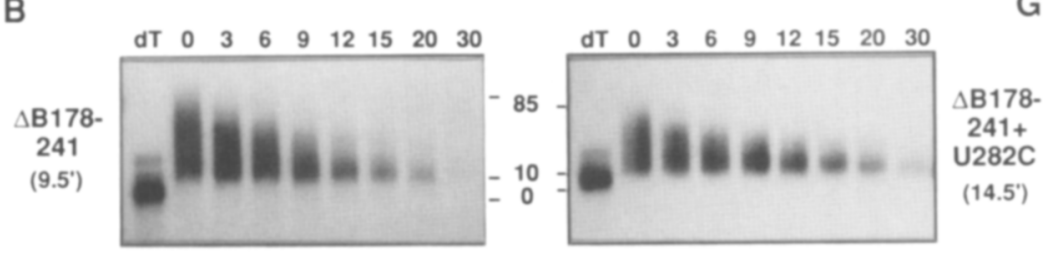

C

C
U248C+
U251G
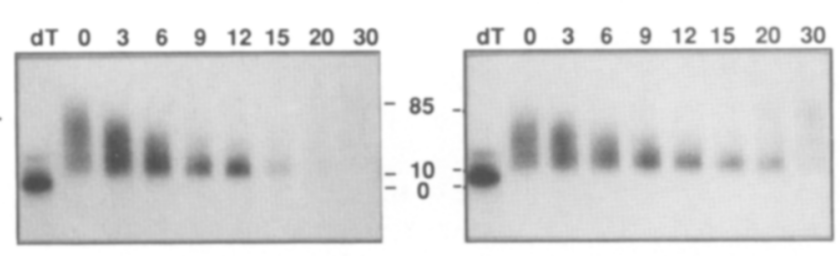

D
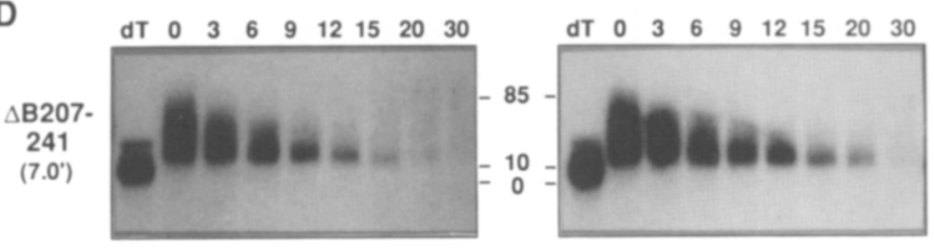

$E$
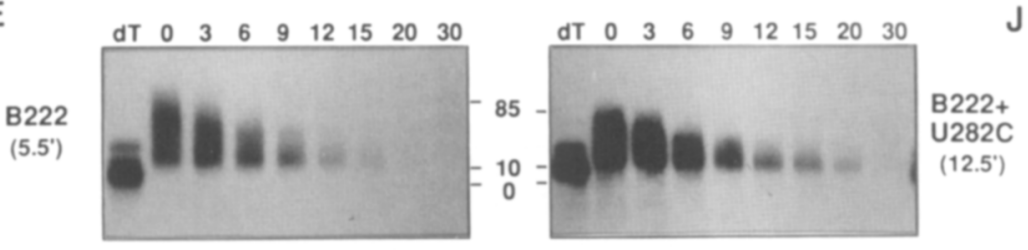

F

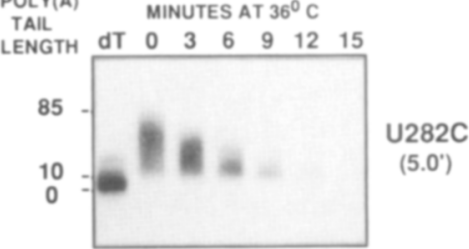

G

H

$\mathrm{U} 248 \mathrm{C}_{+}$

$\mathrm{U} 251 \mathrm{G}+$

U282C

$\left(14.5^{\prime}\right)$

I

$\triangle B 207$ -

$241+$

$\mathrm{U} 282 \mathrm{C}$

$\left(13.5^{\prime}\right)$ sample treated with RNase $\mathrm{H}$ in the presence of oligo(dT) to remove the poly(A) tail. Sizes of transcripts were calculated by comparison to size markers (not shown). The mutations present in the MFA2 transcript are shown on the outside of each panel, along with the half-life of the corresponding transcript. effect of the $\mathrm{U} 282 \mathrm{C}$ point mutation is to increase slightly the persistence of the oligo(A) form (Fig. 6, cf. A and F; U282C $t_{1 / 2}$ of oligo(A) form $=3.9 \pm 0.4 \mathrm{~min}$ (Fig. 6F). When the U282C mutation is combined with mutations that inactivate domain $\mathrm{I}$, however, the stability of the oligo/A) form is increased (Fig. 6, cf. B with G, $\mathrm{C}$ with $\mathrm{H}$, $\mathrm{D}$ with I, and $\mathrm{E}$ with J). Calculation of the half-lives for the oligo(A) species indicates that the effect of the $\mathrm{U} 282 \mathrm{C}$ mutation is an additional two- to threefold increase in the stability of the oligo(A) species [e.g., $\Delta \mathrm{B} 178$ $241+\mathrm{U} 282 \mathrm{C}, t_{1 / 2}$ of oligo(A) form $=16.8 \pm 0.3 \mathrm{~min}$ (Fig. 6G); U248C + U251G + U282C, $t_{1 / 2}$ of oligo(A) form $=14 \pm 2.9 \mathrm{~min}$ (Fig. $6 \mathrm{H}$ ); $\Delta \mathrm{B} 207-241+\mathrm{U} 282 \mathrm{C}, t_{1 / 2}$ of oligo(A) form $=9.5 \pm 1.5 \mathrm{~min}$ (Fig. 6I); B222 + U282C, $t_{1 / 2}$ of oligo(A) form $=8.5 \pm 0.5 \mathrm{~min}$ (Fig. $\left.\left.6 \mathrm{~J}\right)\right]$.

From these observations we conclude that region I can influence the rate of poly $(\mathrm{A})$ shortening and subsequent decay of the oligo(A) form, presumably through removal of the final 10-12 adenylate residues. Region II primarily functions to stimulate the decay of the oligo(A) form. Because region I can normally stimulate both phases of the deadenylation reaction, these results suggest a possible reason why mutation of region II does not affect a transcript with a complete region I.

\section{Discussion}

The 3' UTR contains sequences that promote rapid decay

A combination of genetic and biochemical evidence indicates the existence of two regions within the MFA2 $3^{\prime}$ UTR that are involved in stimulating mRNA decay. Deletions or point mutations within one of these regions, termed region I (nucleotides 184-241), increased the transcript half-life by two- to threefold (Figs. 2, 3, and 4) and indicated the importance of this region. The second region, termed region II (nucleotides 248-288), is not required for rapid decay as evidenced by the rapid decay of transcripts containing either point mutations (U282C; Fig. 5) or deletions $(\Delta \times 245-288$, Fig. 3$)$ in this region. Evidence that this second region is involved in stimulat- 
ing mRNA decay comes from the observation that the addition of the region II mutation U282C to transcripts with region I lesions increases the half-life to a maximum of $\sim 15 \mathrm{~min}$ (Fig. 3 and 5). We interpret these results to argue that although it is not required, region II can also stimulate mRNA turnover, albeit at a reduced rate when compared with region I. In principle, these two regions could be parts of a single functional unit, which can tolerate some alterations without loss of function, or may act independently to affect mRNA decay. In either case, our results indicate that the sequences within the MFA2 $3^{\prime}$ UTR that stimulate decay are at least partially functionally redundant.

\section{Decay of the MFA2 transcript by deadenylation}

What is the role of these elements in stimulating mRNA decay? Our results suggest that these regions function to stimulate two distinct steps in the decay pathway of the $M F A 2$ transcript. The first phase is a rapid deadenylation from the starting poly $(\mathrm{A})$ tail length to $\sim 10-12$ adenosine residues. We refer to this process as poly(A) shortening. The rate of this process on the wild-type MFA2 transcript is $\sim 11-14$ adenosines per minute. This is the fastest rate of poly(A) shortening that we have observed on yeast mRNAs and is two- to sixfold faster than rates for several other yeast mRNAs (C. Decker and R. Parker, in prep.). Our results suggest that one role of the region I sequences is to stimulate poly(A) shortening. The critical observation is that all the deletions or point muta- tions that affect the ability of region I to stimulate mRNA degradation also decrease the rate of loss of transcripts with long poly(A) tails (Fig. 6). Although these experiments do not rule out the formal possibility that all of these mutations have affected overlapping, but independent sequences, stimulating both deadenylation and mRNA degradation, the simplest interpretation of this result is that the mutations that affect mRNA decay rate do so by affecting the rate of deadenylation. Consistent with this model, we have recently shown, by examining the deadenylation and degradation of a synchronously transcribed population of MFA2 transcripts, that transcripts with long poly(A) tails are deadenylated to the oligo(A) form before any mRNA degradation occurs (C. Decker and R. Parker, in prep.). These results led us to propose that the first step in the decay pathway for this mRNA is the shortening of the poly $(\mathrm{A})$ tail to an oligo(A) form (Fig. 7) and that altering the rate of this reaction will change the overall decay rate of the transcript.

What is the fate of the oligo(A) form of the MFA2 transcript? Although we are not able to rule out the fact that this species is degraded directly without further deadenylation (Fig. 7, right pathway), our results favor a pathway in which the final adenylate residues are removed before the body of the transcript is degraded (Fig. 7, left pathway). We refer to this process as terminal deadenylation. The key observations supporting this model are that all of the mutations within region I that affect the initial poly(A) shortening rate also affect the stability of
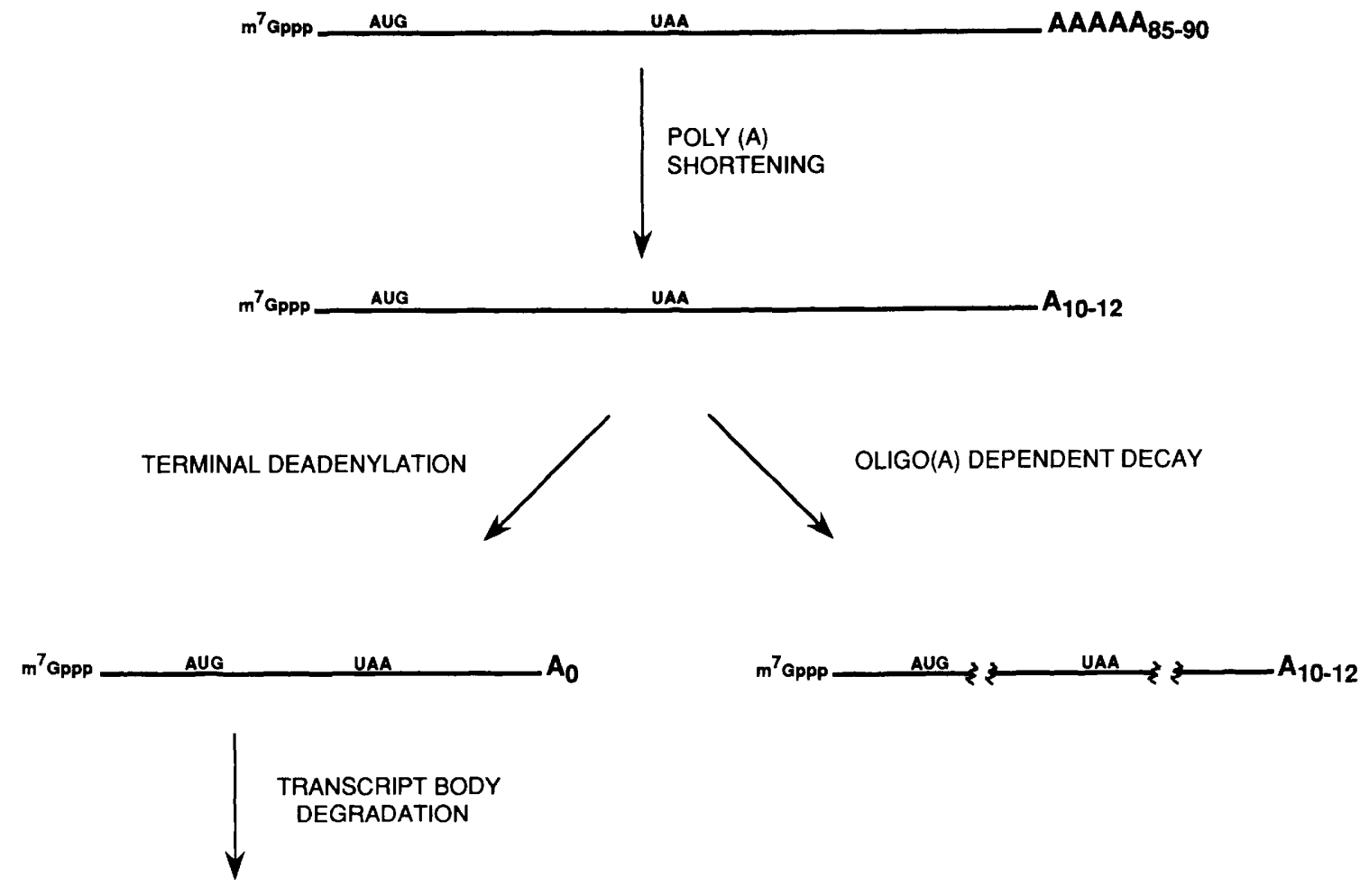

Figure 7. Proposed decay pathway for the MFA2 transcript. 
the oligo(A) form (Fig. 6). Thus, the sequence requirements for the stimulation of the rate of poly $(\mathrm{A})$ shortening are, at least in part, the same for stimulating the decay of the oligo(A) form. The simplest explanation for these observations is that the same machinery not only functions to promote the initial shortening of the poly $(\mathrm{A})$ tail but also its terminal deadenylation. Because we have been unable to detect any transcripts in vivo with $<10-$ 12 adenylate residues, the mechanism of the decay of the body of the message is unknown but must be extremely rapid and conceivably could be coupled to a final deadenylation event.

Interaction between the MFA2 3' UTR and the deadenylation machinery

A first step in understanding the interactions that allow 3 ' UTR sequences to stimulate the deadenylation process will be to determine the features of the $3^{\prime}$ UTR that are recognized by the mRNA deadenylation/turnover machinery. Our deletion analysis demonstrated that although the entire sequence contained within region I is required for optimal stimulation of decay, there is some functional redundancy of these sequences (Fig. 3). This feature is highlighted by the summation of all our point mutations where we have altered 29 nucleotides within the 3' UTR, either individually or in various combinations with only minimal effect (less than twofold) on mRNA decay. Moreover, the few point mutations that do have some effect by themselves, U215A, A250G, and the combination, U248C + U251G, can all be interpreted as allowing novel secondary structures to form and highlight the pitfalls of a detailed interpretation of mutations without structural information. These results raise the possibility that there is no simple unique binding site for an mRNA turnover factor in the MFA2 3' UTR and suggest that there are either multiple redundant sites or the critical feature is an overall structural feature (e.g., being single stranded) that is unaffected by most point mutations.

One notable feature of the MFA2 3' UTR is six repeats of a sequence motif consisting of a pyrimidine run followed by the trinucleotide CAU (underlined in Fig. 4). The observation that the U282C mutation is within one of the region II repeats raises the possibility that these sequences are functionally important. If this sequence motif is able to stimulate the final deadenylation process, the presence of a copy within region I may explain why region II mutations by themselves do not affect mRNA half-life. A corollary of this model is that either the six copies within the MFA2 3' UTR are not functionally equivalent, owing to spacing or sequence, or more than one repeat is required for terminal deadenylation in vivo. Further experiments should clarify these points.

What proteins could be involved in this process? In the simplest model, such interactions could occur directly with two proteins known to play a role in mRNA deadenylation, the poly $(\mathrm{A})$-binding protein $(\mathrm{PAB})$, which is known to be required for poly(A) tail shortening in yeast (Sachs and Davis 1989) or the poly(A) nuclease (PAN) that degrades poly $(\mathrm{A})$ tails in a PAB-dependent manner (Lowell et al., this issue). Interestingly, in a partially purified system, the PAN nuclease also exhibits two distinct phases of deadenylation that may be analogous to the poly $(\mathrm{A})$ shortening and terminal deadenylation seen in vivo (Lowell et al., this issue). Given that different RNA-binding domains within $\mathrm{PAB}$ can, and sometimes prefer to, bind other RNAs besides poly(A) (e.g., Burd et al. 1991), one possible model is that features within the MFA2 3' UTR stimulate deadenylation by providing additional PAB sites. Such a structure may represent an optimal substrate for PAN. Alternatively, an additional component that aids in the assembly of a deadenylation complex may be involved. In this latter view, the deadenylation machinery would be more complex and might be analogous to the enzyme complex involved in adenylation (for review, see Wickens 1990). This type of component might be similar to proteins recently identified in mammalian cells that bind to the AU-rich destabilizing sequences and lead to accelerated mRNA turnover (Brewer 1991; Vakalopoulou et al. 1991).

\section{mRNA decay and deadenylation}

How general is a mRNA decay pathway that is initiated by deadenylation? Recent experiments suggest that at least two other yeast mRNAs require deadenylation to the oligo(A) form before mRNA decay initiates (C. Decker and R. Parker, in prep.). Moreover, it should be noted that the pathway we have proposed for the MFA2 mRNA is similar to that described previously for the mammalian mRNAs encoded by the c-myc and c-fos genes. In these cases in vivo and in vitro experiments argue that the decay of these mRNAs is preceded by deadenylation (Brewer and Ross 1988; Shyu et al. 1991; Swartwout and Kinniburgh 1991). These observations indicate that deadenylation as a mechanism of mRNA decay is conserved from yeast to mammals. Because essentially all mRNAs are subject to cytoplasmic poly(A) shortening, this pathway is likely to have an impact on the decay of a substantial number of mRNAs.

Our results with the MFA2 transcript suggest that there are two distinct phases to the deadenylation-decay process, an initial shortening of the poly(A) tail to an oligo(A) form and a subsequent terminal deadenylation step. Other experiments suggest that the presence of two distinct phases in the deadenylation process is a common feature for many mRNAs (e.g., Mercer and Wake 1985; Restifo and Guild 1986). For example, in the case of the c-myc mRNA, the transcript has been seen to pass through a poly(A) $)^{-}$pool as defined by binding to oligoldT)-cellulose (Swartwout and Kinniburgh 1991). Because mRNAs with oligo(A) tails will bind only poorly to oligo(dT)-cellulose (Groner et al. 1974), this poly(A) ${ }^{-}$ pool might be analogous to the oligo(A) form that we observe with the MFA2 transcript. Similarly, mutations have been identified in the c-fos AU-rich instability element that do not affect the rate of deadenylation but do stabilize a form of the transcript with, at most, an oligo(A) tail (Shyu et al. 1991). 
One implication of these results is that the stability of a transcript subject to this decay pathway could be affected by altering either the rate and/or the extent of poly(A) shortening or the rate of terminal deadenylation. In this light, it should be noted that the sequences within the MFA2 3' UTR are not required for poly(A) shortening but serve to stimulate the rate of this reaction. This feature may explain why the maximum halflife that we observed for any MFA2 transcript was 15 min, despite the fact that many yeast mRNAs can have half-lives of $>45 \mathrm{~min}$ (Herrick et al. 1990). Although it is possible that this intermediate decay rate arises from other features of the MFA2 transcript that stimulate alternative decay pathways, a half-life of 15 min may reflect the unstimulated, or default, rate of deadenylation and mRNA turnover. A corollary of this view is that extremely stable mRNAs may contain features that inhibit one or more steps in the deadenylation process. Such features might be the explanation for the extreme stability of some deadenylated or partially deadenylated mRNAs in both mammals (e.g., Krowczynska et al. 1985; Shyu et al. 1991) and in yeast (Herrick et al. 1990; C. Decker and R. Parker, in prep.). Given the growing consensus that the metabolism of poly(A) tails plays an important role in regulating gene expression both at the level of translation and transcript stability (for review, see Jackson and Standart 1990; Sachs 1990), it will be important to understand the features of mRNAs that regulate poly(A) tail length and to determine the gene products that regulate this process.

\section{Materials and methods}

Synthetic yeast medium lacking tryptophan $(-\mathrm{TRP})$ was prepared by standard techniques and used to maintain and select for the plasmids containing the MFA2 gene and mutants. Oligonucleotides were synthesized by the Arizona Research Laboratory Biotechnology Facility.

\section{In vivo screen for MFA2 overexpression mutants}

We used polymerase chain reaction (PCR) mutagenesis coupled with in vivo recombination (Muhlrad et al. 1992) to generate a collection of point mutations within the MFA2 gene to screen for overexpressing mutants. The starting plasmid for our PCR mutagenesis, pRP264, was constructed by inserting an EcoRIHindIII (filled-in) fragment from pSM29 (Michaelis and Herskowitz 1989) containing the MFA2 gene into the SphI (filled in with Klenow fragment--EcoRI sites of a yeast centromere plasmid, pUN0 (Elledge and Davis 1988), containing the TRP1 gene. During construction, the final $5.3-\mathrm{kb}$ plasmid pRP264 lost the EcoRI cloning site. The yeast strain used in the screen was SM1229 (MATa; trp1- $\Delta 1$; leu2-3,112; ura3-52; his4-539; can1; MFA1::LEU2; MFA2::URA3; Michaelis and Herskowitz 1989).

Two pools of mutants were screened. In one, the entire MFA2 gene was amplified with standard sequencing primers located within the polylinker sequences flanking the MFA2 sequence and used to repair a gap between the SphI site located $5^{\prime}$ of the $M F A 2$ gene and the BamHI site located beyond the $3^{\prime}$ end of the gene. Screening $\sim 200$ recombinants from this pool yielded 59 null mutants in MFA2 and 2 mutants that yielded larger halos. Of these mutants, only one, termed M5, affected the mRNA decay rate (see Results). In a second screen, the MFA2 gene was amplified with a $5^{\prime}$ primer, oRP61, located just upstream of the AUG start codon and a $3^{\prime}$ primer in the polylinker sequences and used to repair a gap between the two BamHI sites in MFA2. To reduce the extent of mutagenesis, we decreased the concentration of $\mathrm{Mn}^{2+}$ in these reactions to $0.125 \mathrm{~mm}$. Screening $\sim 2000$ recombinants from this pool yielded 51 null mutants and 9 mutants that gave rise to larger halos. Of these 9, only 2, U240G and U215A, affected the mRNA decay rate. Halo assays were performed as follows: A lawn of cells (SM1086: MAT $\alpha_{\text {; }}$ sst 2 ; rme; his6; met1; can1; cyh2; $10^{6}$ cells in $2.5 \mathrm{ml}$ of complete $2 \%$ top agar) was poured onto a complete plate and allowed to solidify and dry; patches of MATa cells were then replica plated onto it. After incubation at $24^{\circ} \mathrm{C}$ or $30^{\circ} \mathrm{C}$ for $24-48$ hr halos were scored by size comparison to strains containing both wild-type and overexpression MFA2 plasmids la $2 \mu$ version, pSM25; Michaelis and Herskowitz 1989).

Plasmids from overexpressing strains were extracted from yeast (Sherman et al. 1986), and reintroduced into the strain yRP384 (MATa; ura3-52; leu2-3, -112; trp1- $\Delta 1$; his3- $\Delta 200$; MFA1::LEU2; MFA2::URA3; rpb1-1). This strain, which is deleted for both a-factor genes and has the $r p b 1-1$ temperaturesensitive mutation in RNA polymerase II to allow measurement of mRNA decay rates, was constructed by a cross between

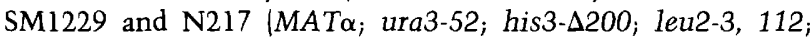
rpb1-1; ade2). Overexpression phenotypes were confirmed in this strain by halo assays, as well as a dilution assay (Michealis and Herskowitz 1989). In.all cases, the plasmids reproduced the phenotypes (data not shown).

The entire $M F A 2$ gene from these mutants was sequenced using standard techniques with a collection of specific primers.

\section{Oligomutagenesis}

To create the plasmid for oligomutagenesis, pRP270, the 2.0-kb HindIII fragment of pUN30 (Elledge and Davis 1988) containing the phage M13 origin of replication was inserted into the HindIII site of pRP264. Site-directed mutagenesis was performed by standard methods. After mutagenesis, the entire $M F A 2$ gene was sequenced for any new mutation before analysis in vivo.

\section{Construction of deletions in the MFA2 3' UTR}

The $\Delta \mathrm{X} 245-288$ deletion, plasmid pRP323, was constructed by digesting plasmid pRP322, which contains an introduced $X$ hoI site, X245 (see Fig. 4), with XhoI, filling with Klenow, and cutting with $B g I I I$, yielding a $3.7-\mathrm{kb}$ fragment. This fragment was then ligated to a 2.3-kb NdeI (filled-in)-BglII fragment from pRP270. This deletion removes 41 bp of the MFA2 3' UTR.

The $\triangle \mathrm{B} 178-241$ deletion, pRP324, was constructed by digesting plasmid pRP320, which contains the $5^{\prime}$-most BgIII site created by site-directed mutagenesis, B178, with BgIII and a $3.7-\mathrm{kb}$ fragment purified. This fragment was then ligated to a $2.3-\mathrm{kb}$ BglII fragment from pRP321, which contains the $3^{\prime}$-most BglII site introduced by mutagenesis, B241. This deletion removes 60 bp of the 3' UTR of MFA2. The U282C point mutation was added to the $\triangle \mathrm{B} 178-241$ deletion, resulting in plasmid pRP361, by cutting pRP324 with BglII and purifying the $3.7-\mathrm{kb}$ fragment. This fragment was then ligated to the 2.3-kb BglII fragment from plasmid pRP329, which contains the 3 '-most BglII site, $\mathrm{B} 241$, and the U282C point mutation (introduced by oligomutagenesis).

The $\Delta \mathrm{B} 178-288$ deletion, pRP325, was constructed by digesting pRP320 with $B g I I I$ and purifying the $3.7-\mathrm{kb}$ fragment. This fragment was then ligated to a $2.25-\mathrm{kb} N d e \mathrm{I}$ (filled in, followed 
by the addition of a BglII linker)-BglII fragment from plasmid $\mathrm{pRP} 270$. This construction deletes a total of $115 \mathrm{bp}$ in the $3^{\prime}$ end of MFA2.

Deletions between the $5^{\prime}$-most BglII site, B178, and the BglII sites B207 and B222, with or without the U282C lesion, were created by purifying the $550-b p$ SphI-BglII fragment from pRP320. This fragment was then inserted into SphI-BglII-cut vectors purified from pRP370 (B207) yielding pRP374 (29-nucleotide deletion), pRP371 (B207 + U282C) yielding pRP375 (29nucleotide deletion), pRP372 (B222) yielding pRP376 (44-nucleotide deletion), and pRP373 (B222 + U282C) yielding pRP377 (44-nucleotide deletion).

Deletions between the 3 '-most BgIII site, B241, and the B207 site, with or without the U282C lesion, were created by first purifying the 700-bp Bg1II-NarI fragment from pRP32l (containing the B241 site). This fragment was then inserted into BgIII/NarI-cut vectors purified from pRP370 (B207) yielding pRP378 (34-nucleotide deletion), and pRP371 (B207 + U282C) yielding pRP379 (34-nucleotide deletion).

All plasmid constructions and mutations were confirmed by diagnostic restriction digests and/or sequencing.

\section{RNA analysis}

mRNA preparation and analysis was done essentially as previously described (Herrick et al. 1990; Parker et al. 1991). Analysis of poly(A) tail lengths was performed by running $8 \mu \mathrm{g}$ of total RNA on a $6 \%$ polyacrylamide $/ 7 \mathrm{M}$ urea gel that was electroblotted and probed by standard techniques. RNase $\mathrm{H}$ reactions were done as described previously (Donis-Keller 1979).

\section{Acknowledgments}

We thank Alan Sachs for communication and discussion of results before publication, and Carolyn Decker, Alison Adams, and John Little for helpful comments on the manuscript. This work was supported by grants to R.P. from the Chicago Community Trust and the National Institutes of Health (GM45443).

The publication costs of this article were defrayed in part by payment of page charges. This article must therefore be hereby marked "advertisement" in accordance with 18 USC section 1734 solely to indicate this fact.

\section{References}

Binder, R., S.L. Hwang, R. Ratnasabapathy, and D.L. Williams. 1989. Degradation of apolipoprotein II mRNA occurs via endonucleolytic cleavage at 5'-AAU-3'/5'-UAA-3' elements in single stranded loop domains of the $3^{\prime}$ noncoding region. $I$. Biol. Chem. 264: 16910-16918.

Brewer, G. 1991. An A + U-rich element RNA-binding factor regulates c-myc mRNA stability in vitro. Mol. Cell. Biol. 11: $2460-2466$.

Brewer, G. and J. Ross. 1988. Poly|A) shortening and degradation of the 3' A + U-rich sequences of human c-myc mRNA in a cell free system. Mol. Cell. Biol. 8: 1697-1708.

Brown, B. and R. Harland. 1990. Endonucleolytic cleavage of a maternal homeo box mRNA in Xenopus oocytes. Genes \& Dev. 4: 1925-1935.

Burd, C.G., E.L. Matunis, and G. Dreyfuss. 1991. The multiple domains of the mRNA poly(A)-binding protein have different RNA-binding activities. Mol. Cell. Biol. 11: 3419-3424.

Cleveland, D. 1989. Gene regulation through messenger RNA stability. Curr. Opin. Cell Biol. 1: 1148-1153.

Donis-Keller, H. 1979. Site specific enzymatic cleavage of RNA. Nucleic Acids Res. 7: 179-192.
Elledge, S.J. and R.W. Davis. 1988. A family of versatile centromeric vectors designed for use in the sectoring-shuffle mutagenesis assay in Saccharomyces cerevisiae. Gene 70: 303312.

Groner, B., N. Hynes, and S. Phillips. 1974. Length heterogeneity in the poly(adenylic acid) region of yeast messenger ribonucleic acid. Biochemistry 13: 5378-5383.

Herrick, D., R. Parker, and A. Jacobson. 1990. Identification and comparison of stable and unstable mRNAs in Saccharomyces cerevisiae. Mol. Cell. Biol. 10: 2269-2284.

Higgins, C.F. 1991. Stability and degradation of mRNA. Curr. Opin. Cell Biol. 3: 1013-1018.

Jackson, R.J. and N. Standart. 1990. Does the poly(A) tail and 3' untranslated region control mRNA translation? Cell 62: 1524.

Krowczynska, A., R. Yenofsky, and G. Brawerman. 1985. Regulation of messenger RNA stability in mouse erythroleukemia cells. J. Mol. Biol. 181: 231-239.

Mercer, J.F.B. and S.A. Wake. 1985. An analysis of the rate of metallothionein mRNA poly(A)-shortening using RNA blot hybridization. Nucleic Acids Res. 13: 7929-7943.

Michaelis, S. and I. Herskowitz. 1989. The a-factor pheromone of Saccharomyces cerevisiae is essential for mating. Mol. Cell. Biol. 8: 1309-1318.

Muhlrad, D., R. Hunter, and R. Parker. 1992. A rapid method for the localized mutagenesis of yeast genes. Yeast 8: 79-82.

Parker, R. and A. Jacobson. 1990. Translation and a forty-two nucleotide segment within the coding region of the mRNA encoded by the MAT 1 gene are involved in promoting mRNA decay in yeast. Proc. Natl. Acad. Sci. 87: 2780-2784.

Parker, R., S. Peltz, D. Herrick, and A. Jacobson. 1991. Measurement of mRNA decay rates in Saccharomyces cerevisiae. Methods Enzymol. 194: 415-422.

Peltz, S.W., G. Brewer, P. Berstein, P. Hart, and J. Ross. 1991. Regulation of mRNA turnover in eukaryotic cells. Crit. Rev. Eukaryotic Gene Expression. 1: 99-126.

Restifo, L.L. and G.M. Guild. 1986. Poly(A) tail shortening of coregulated transcripts in Drosophila. Dev. Biol. 115: 507510.

Sachs, A. 1990. The role of poly(A) in the translation and stability of mRNA. Curr. Opin. Cell. Biol. 2: 1092-1098.

Sachs, A.B. and R.W. Davis. 1989. The poly(A) binding protein is required for poly (A) shortening and $60 \mathrm{~S}$ ribosomal subunitdependent translation initiation. Cell 58: 857-867.

Sherman, F., G.R. Fink, and J.B. Hicks. 1986. Laboratory course manual for methods in yeast genetics. Cold Spring Harbor Laboratory, Cold Spring Harbor, New York.

Shyu, A.-B., J.G. Belasco, and M.E. Greenberg. 1991. Two distinct destabilizing elements in the c-fos message trigger deadenylation as a first step in rapid mRNA decay. Genes \& Dev. 5: 221-234.

Stormo, G.D. 1986. Translational Initiation. In Maximizing gene expression (ed. W. Reznikoff and L. Gold), pp.195-224. Butterworths, Boston, MA.

Swartwout, S.G. and A.J. Kinniburgh. 1991. c-myc mRNA degradation in growing and differentiating cells: Possible alternative pathways. Mol. Cell. Biol. 9: 288-295.

Vakalopoulou, E., J. Schaack, and T. Shenk. 1991. A 32-kilodalton protein binds to AU-rich domains in the $3^{\prime}$ untranslated regions of rapidly degraded mRNAs. Mol. Cell. Biol. 11: 3355-3364.

Wickens, M. 1990. How the messenger got its tail: Addition of poly(A) in the nucleus. Trends Biochem. Sci. 15: 277-281.

Wilson, T. and R. Triesman. 1988. Removal of poly(A) and consequent degradation of the c-fos mRNA facilitated by $3^{\prime} \mathrm{AU}-$ rich sequences. Nature 336: 396-399. 


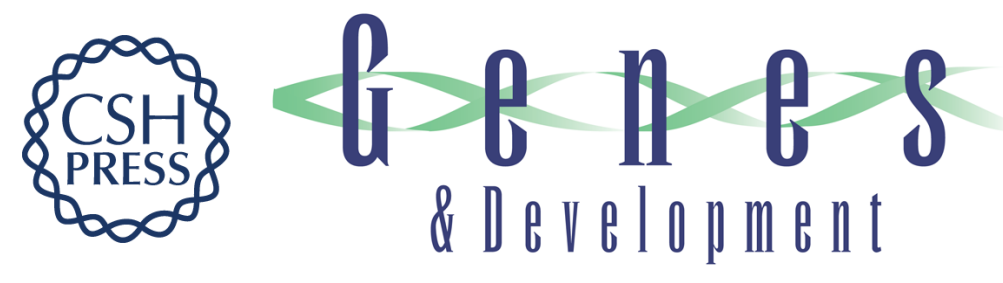

\section{Mutations affecting stability and deadenylation of the yeast MFA2 transcript.}

D Muhlrad and R Parker

Genes Dev. 1992, 6:

Access the most recent version at doi:10.1101/gad.6.11.2100

References This article cites 27 articles, 9 of which can be accessed free at: http://genesdev.cshlp.org/content/6/11/2100.full.html\#ref-list-1

License

Email Alerting

Receive free email alerts when new articles cite this article - sign up in the box at the top Service right corner of the article or click here.

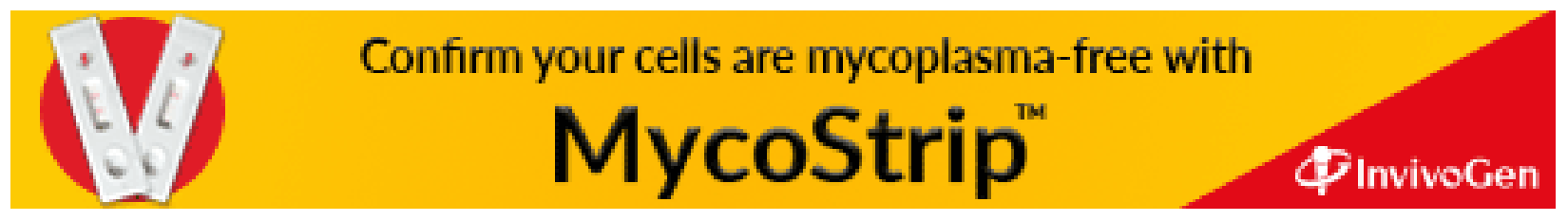

